\title{
GPS L5 Signal Acquisition and Tracking under Unintentional Interference or Jamming
}

\author{
Ilir F. Progri \\ Giftet Inc., 5 Euclid Ave. \#3, Worcester, MA 01610, USA \\ ORCID: 0000-0001-5197-1278
}

Correspondence should be addressed to Ilir Progri; iprogri@giftet.com

Received March 23, 2017; Revised March 24, 31, 2017-June 7, 2017, Accepted July 16, 2017; Published November 1, 2017.

Scientific Editor-in-Chief/Editor: Ilir F. Progri

Copyright (C) 2017 Ilir F. Progri. This is an open access article distributed under the Creative Commons Attribution License, which permits unrestricted use, distribution, and reproduction in any medium, provided the original work is properly cited.

The GPS signal consists of several signals transmitted at the $\mathrm{L}_{1}, \mathrm{~L}_{2}$, and $\mathrm{L}_{5}$ carrier frequencies. The signal at $\mathrm{L}_{1}=$ 1575.42 MHz and $\mathrm{L}_{2}=1227.6 \mathrm{MHz}$ consists of the civilian C/A code signals with pseudorandom sequences of length 1023 and chipping frequency of $1.023 \mathrm{MHz}$ and the $\mathrm{P}(\mathrm{Y})$ code signals of length 1 week and chipping frequency of 10.23 MHz. The new L5 signal is QPSK-modulated, centered on 1176.45 MHz. Its two components have each a different spreading code at chipping frequency of 10.23 MHz. The in-phase component carries the navigation message, at 100 symbols per second ( 50 bits per second with a convolutional encoder) while the quadrature component, called the pilot channel, carries no message at all. Most GPS receivers, whether military or civilian, are designed in a way that the acquisition of the $\mathrm{P}(\mathrm{Y})$ code depends on the acquisition and tracking of the C/A code. Moreover, if the acquisition of the $\mathrm{P}(\mathrm{Y})$ code is lost, due to reasons that are explained in the paper, then the reacquisition of the $\mathrm{P}(\mathrm{Y})$ code will again depend on the acquisition and tracking of the C/A code. Therefore, for most commercially available receivers, the reliable acquisition and tracking of the C/A code is critical for the entire operation of a GPS receiver.

However, it is well-known the vulnerability of the GPS C/A code signal acquisition to unintentional interference or jamming. The main theme of this paper is the reexamination of the GPS C/A code signal acquisition and tracking under the situation of the unintentional interference or jamming. A variety of jamming signals are considered such as: periodic, deterministic; aperiodic, deterministic; and noisy type signals or non-deterministic.

However, the majority of vendors are still producing correlator type receivers. As shown by the simulation results of this paper and other previous publications a sliding correlator type of receiver used to acquire and track the C/A code or $\mathrm{L}_{5}$ code can be easily jammed by a similar $\mathrm{C} / \mathrm{A}$ code replica or $\mathrm{L}_{5}$ code replica at power levels $10 \mathrm{~dB}$ or higher above noise power. GAMES, GILS, and GIANT are not the most economically viable solutions in the market to handle GPS protection of the civilian receivers. A maximum likelihood GPS receiver, which considers all the signals in the environment, can be used to acquire and track successfully the C/A and the $\mathrm{L}_{5}$ GPS signals when the jamming signal power are in the range or $10 \mathrm{~dB}$ or higher above the noise power. In summary, the direction of designing acquisition and tracking receivers for future $\mathrm{C} / \mathrm{A} \mathrm{L}_{1}$ code or $\mathrm{L}_{5}$ GPS signals should go towards joint signal acquisition and tracking. There are three main challenges with these types of receivers: algorithm complexity, computational 
power, and test with real data. All these remain to be studied and analyzes further in the future. I think it is going to be a breakthrough in the state of the art of GPS receivers when these algorithms are ultimately implemented and a lot more work is required to arrive at that point which some of it is going to be published in future publications.

Index Terms - GPS, $\mathrm{L}_{1}, \mathrm{~L}_{2}, \mathrm{~L}_{5}, \mathrm{C} / \mathrm{A}$ code, $\mathrm{P}(\mathrm{Y})$ code, signal acquisition, signal tracking, unintentional interference, jamming, non-deterministic.

\section{Introduction}

In the last decade the GNSS community is heavily involved in the analysis, design, development, and implementation of GPS systems that operate at the $\mathrm{L}_{5}=1176.45 \mathrm{MHz}$ frequency [1][11].

There are several advantages of the new GPS signal. It has stronger power compared to the civilian signals transmitted at the $\mathrm{L}_{1}=1575.42 \mathrm{MHz}$ and $\mathrm{L}_{2}=1227.6 \mathrm{MHz}$ [2]. Unlike the Binary Phase Shift Keying (BPSK) modulation scheme used for the civilian signals transmitted at the $\mathrm{L}_{1}$ and $\mathrm{L}_{2}$, the modulation scheme used for the new $\mathrm{L}_{5}$ signal is Quadraphase Shift Keying (or QPSK). The chipping frequency for the new signal is 10 times higher than chipping frequency used for the signals in the $L_{1}$ and $L_{2}$. It appears to offer better multipath and narrowband interference resistance [7]. For these reasons and other reasons reported in the literature the new signal is more suitable for a variety of civilian applications in addition to the $\mathrm{L}_{1}$ and $\mathrm{L}_{2}$ signals.

Acquisition algorithms, multipath mitigation performance receiver implementation issues, and software receiver approaches of the GPS L 5 signal are discussed in [3]-[9], [11].

Recently, two techniques are proposed as interference mitigation means for $\mathrm{L}_{5}$ GPS receivers [5], [9]. This does not exclude all the interference mitigation techniques proposed for the GPS $\mathrm{L}_{1}$ and the $\mathrm{L}_{2}$ receivers.

First, Bastide el al [5] considers assessment of $\mathrm{L}_{5}$ receiver performance in presence of interference using a realistic receiver simulator. According to Bastide el al [5] $\mathrm{L}_{5}$ band is expected to face a strong interference environment mainly because of pulsed DME/TACAN signals. Moreover Bastide el al [5] has developed an $\mathrm{L}_{5}$ signal generator and receiver simulator under Labview environment which enables to understand the tracking performance in normal conditions and different types of interferers. These types of interferers are: Continuous wave interference $(\mathrm{CW})$; Frequency Modulation (FM) interference; DME $\backslash$ TACAN pulsed signals. More discussion about Bastide el al [5] is found further in the paper.
Second, Issler et al [10] propose a probabilistic approach of frequency diversity as interference mitigation means. According to Issler et al [10] frequency diversity is a means to mitigate jamming and interference. Moreover Issler et al [10] provide an elegant description of an aperiodic deterministic jammer; namely, a military radar.

The paper is organized as follows. First, the $\mathrm{L}_{5}$ signal model is presented. Second, the $\mathrm{L}_{5}$ signal acquisition and tracking are considered. Third, we propose three types of jamming signal: periodic, deterministic; a periodic, deterministic; and noisy type signals or non-deterministic. Fourth, we analyze the acquisition and tracking performance based on a MATLAB ${ }^{\circledR}$ simplified model under a nosy type jamming signal. Fifth, some conclusions about the effect of these types of jamming signals on the $\mathrm{L}_{5}$ acquisition and tracking performance are presented

As expected the noisy type of interference or nondeterministic signals will impose greater threat to the $\mathrm{L}_{5}$ acquisition and tracking performance. Several kinks of diversities can be employed in order to mitigate these types of interference signals. These kinds of diversities would be for example code; frequency; antenna; and space. The first three kinds of diversities are currently well known and well understood in the community and implemented in the signal structure $^{\mathrm{i}}$ and depicted in the GNSS signal design ${ }^{\mathrm{ii}}$ [15]-[25]. And to a large extent the GPS system is employing these kinds of diversities. However, the space diversity approach is still not well understood and therefore, it remains to be determined that that technique may offer some benefits to the community. Moreover, more sophisticated receiver acquisition and tracking algorithms must be developed and implemented one of which is discussed in our previous papers [14].

\section{$2 \quad$ L5 Signal Model and Transmitter Design ${ }^{\text {iii }}$}

A block diagram of the GPS $\mathrm{L}_{5}$ signal, $s_{k}\left(\omega_{k}, t\right)$, is depicted in Fig.1(a). As shown in Fig.1(a) the modulation used for the $\mathrm{L}_{5}$ signal is QPSK; hence, there are two orthogonal carrier components: the in-phase (I) component, $\cos \left(\theta_{k}\right)$, and the 
quadrature $(\mathrm{Q})$ component, $\sin \left(\theta_{k}\right)$, as illustrated in Fig.1(b) where $\theta_{k}=\omega_{k} t+\phi_{k}$ is the total carrier phase and $\omega_{L 5}=$ $2 \pi f_{L 5}(\mathrm{rad} / \mathrm{sec}), f_{L 5}=1176.45 \mathrm{MHz}$ is the GPS $\mathrm{L}_{5}$ signal frequency, and $\phi_{k}$ is the oscillator phase offset.

Two separate bit trains, namely the $\mathbf{w}_{I k}$ and $\mathbf{w}_{Q k}$ sequences, are BPSK modulated with each carrier component respectively. This is accomplished first by converting digital sequences $\mathbf{w}_{I k}$ and $\mathbf{w}_{Q k}$ into analog signals $s_{I k}(t)$ and $s_{Q k}(t)$

$$
\begin{aligned}
& s_{I k}(t)=w_{I k} h\left(t-m T_{c}\right) \\
& s_{Q k}(t)=w_{Q k} h\left(t-m T_{c}\right)
\end{aligned}
$$

where $h(t)$ is a raised cosine filter and second, the GPS $\mathrm{L}_{5}$ signal, $s_{k}\left(\omega_{k}, t\right)$, is given by

$$
s_{k}\left(\omega_{k}, t\right)=G\left[s_{I k}(t) \cos \left(\theta_{k}\right)+s_{Q k}(t) \sin \left(\theta_{k}\right)\right]
$$

where $G=\sqrt{2 E_{c}}$ is the amplifier gain of the carrier and $E_{c}$ is the energy per symbol.

The $\mathbf{w}_{I k}$ and $\mathbf{w}_{Q k}$ sequences are obtained by modulo 2 add of a constant binary sequence, $\mathbf{q}=\{00,01,11,10\}$, which modulates the phase of the $\mathrm{I}_{5}$ and $\mathrm{Q}_{5}$ digital component sequences $\mathbf{z}_{I k}$ and $\mathbf{z}_{Q k}$ respectively:

$$
\mathbf{w}_{i k}=\mathbf{z}_{i k} \oplus \mathbf{q} ; i=\{I, Q\}
$$

The in-phase (I) digital component, $\mathbf{z}_{i k}$, is the modulo-2 sum of three bits: the $I_{5}$ spreading sequence, $\mathbf{a}_{I k}=\left\{a_{I k m}\right\}$, NAV data, $\mathbf{d}_{I k}=\left\{d_{I k m}\right\}$, and a 10-symbol Neuman-Hoffman sequence, $\mathbf{b}_{10 k}=\left\{b_{10 m}\right\}$, as illustrated in Fig. 1(a).

The quadrature (Q) digital component, $\mathbf{z}_{Q k}$, consists of the modulo-2 sum of two bits the $\mathrm{Q}_{5}$ spreading sequence, $\mathbf{a}_{Q k}=$ $\left\{a_{Q k m}\right\}$, and 10-symbol Neuman-Hoffman sequence, $\mathbf{b}_{20 k}=$ $\left\{b_{20 m}\right\}$.

We can write the I and Q components in compact form as follows

$$
\mathbf{z}_{i k}=\mathbf{a}_{i k} \oplus \mathbf{b}_{i k} \oplus \mathbf{d}_{i k} ; i=\{I, Q\}
$$

Where

$$
\begin{aligned}
& \mathbf{b}_{I k} \equiv \mathbf{b}_{10 k}=\left\{b_{10 m}\right\} ; \mathbf{b}_{Q k} \equiv \mathbf{b}_{20 k}=\left\{b_{20 m}\right\} \\
& \mathbf{d}_{Q k} \equiv \emptyset ; \text { empty set or no data }
\end{aligned}
$$

The $\mathrm{I}_{5}$ and $\mathrm{Q}_{5}$ spreading sequences, $\mathbf{a}_{I k}=\left\{a_{I k m}\right\}$ and $\mathbf{a}_{Q k}=\left\{a_{Q k m}\right\}$, are generated at 10.23 Mcps and have a period of 10230 chips (or symbols) and so that one period lasts $1 \mathrm{~ms}$ based on two different maximum length sequences, $\mathbf{x}_{a}$ and $\mathbf{x}_{b k}$, as depicted in Fig. 1(c).

The first maximum length sequence, $\mathbf{x}_{a}$, is generated based on the primitive polynomial $\mathbf{p}_{a}=\{11011000000001\}$ and initial state all 1's. The second maximum length sequence, $\mathbf{x}_{b k}$, is generated from the primitive polynomial, $\mathbf{p}_{b k}=$
$\{11000111011011\}$, and initial state $k$. At the end of every 10230 symbols; i.e., every $1 \mathrm{~ms}$, the $\mathbf{x}_{a}$ is reset to all 1 's and the $\mathbf{x}_{b k}$ to its initial state $k$ [11]. The 37 selected $\mathrm{I}_{5}$ and $\mathrm{Q}_{5}$ spreading sequences, $\mathbf{a}_{I k}=\left\{a_{I k m}\right\}$ and $\mathbf{a}_{Q k}=\left\{a_{Q k m}\right\}$, are given in greater detail in [2].

The data sequence, $\mathbf{d}_{I k}=\left\{d_{I k m}\right\}$, is generated as follows. A 24-bit cyclic redundant code is added to every 267 bits of the GPS $\mathrm{L}_{5} \mathrm{NAV}$ to form a 300 bits data message frame generated at $50 \mathrm{bps}$. Then this message is convolutionally encoded with a rate $1 / 2$, constraint length 7 code resulting in a 100 sps symbol stream as shown in Fig. 1(d). Each symbol is synchronized with the synchronization sequence that is a 10-symbol Neuman-Hoffman sequence, $\mathbf{b}_{10}=\left\{b_{10}\right\}$, clocked at the $1 \mathrm{~ms}$ $\mathrm{I}_{5}$ spreading sequence and it is reset every $10 \mathrm{~ms}$. Since the I digital component carries data it is called the data channel in contrast to the Q component which is called the pilot (or dataless) component. The synchronization sequence, $\mathbf{b}_{20}=$ $\left\{b_{20}\right\}$, is a 20 -symbol Neuman-Hoffman sequence clocked at the $1 \mathrm{~ms} \mathrm{Q}_{5}$ spreading sequence period and it is reset every 20 $\mathrm{ms}$ as depicted in Fig. 1(a).

Here we conclude the discussion of the $\mathrm{L}_{5}$ GPS signal structure, architecture, and design and take a look at the $\mathrm{L}_{5}$ GPS receiver signal model.

\section{$3 \quad$ L5 GPS Receiver Signal Model ${ }^{\text {iv }}$}

Since for most of the state of the art GPS receivers process the received signal at the IF frequency (typically at $160 \mathrm{MHz}$ ); therefore, we will start with an expression of the $\mathrm{L}_{5}$ GPS signal at the IF frequency. Consider $K$ GPS satellites in the sky. The received signal, $x(n)$, at discrete time index, $n$, is given by

$$
x(n)=x_{I}(n)+x_{Q}(n)+\varepsilon(n)
$$

where

$$
\begin{aligned}
& x_{i}(n)=\sum_{k=1}^{K} s_{i k}\left(t_{k}\right) c_{i k} ; i=\{I, Q\} \\
& s_{i k}\left(t_{k}\right)=a_{i k}\left(t_{k}\right) d_{i k}\left(\left[t_{k}\right]_{D}\right) b_{i}\left(t_{k}\right) ; i=\{I, Q\} \\
& d_{I k}\left(\left[t_{k}\right]_{D}\right)=d_{k}\left(\left[t_{k}\right]_{D}\right) ; d_{Q k}\left(\left[t_{k}\right]_{D}\right)=1 \\
& b_{I}\left(t_{k}\right) \equiv b_{10}\left(t_{k}\right) ; b_{Q}\left(t_{k}\right) \equiv b_{20}\left(t_{k}\right) \\
& c_{I k}=\cos \left(\theta_{k}\right) ; c_{Q k}=\sin \left(\theta_{k}\right) \\
& \theta_{k}=2 \pi f_{k} t_{k} \\
& t_{k}=n T_{s}-\tau_{k} \\
& {\left[t_{k}\right]_{D}=\left\lfloor t_{k} / D\right\rfloor}
\end{aligned}
$$






(a) A block diagram of a GPS $\mathrm{L}_{5}$ signal generator - taken and modified from [11].

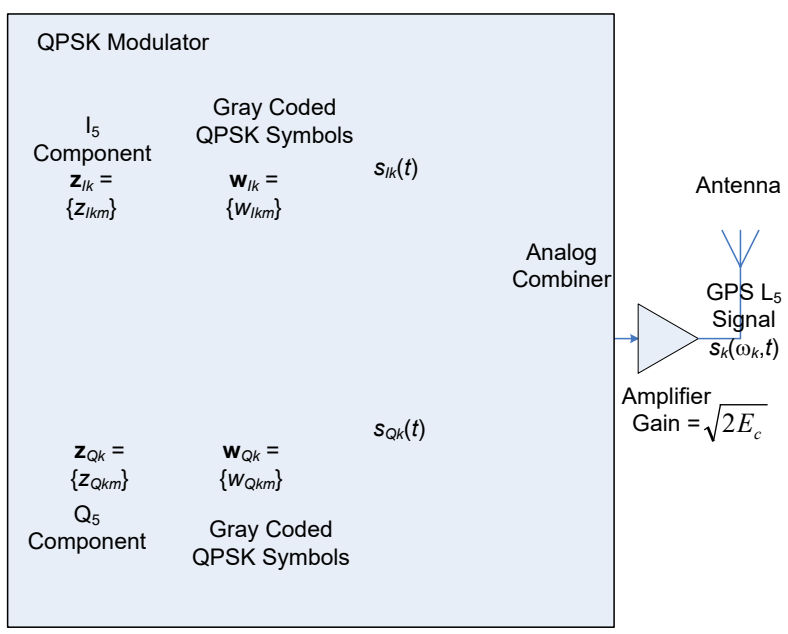

(b) A block diagram of the QPSK modulator and antennataken and modified from [12].

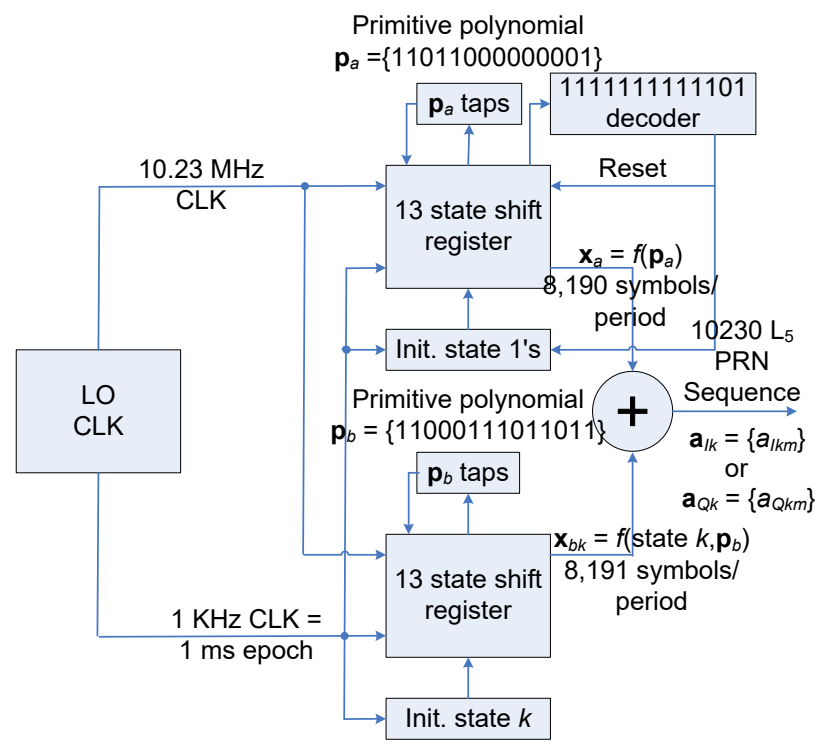

(c) A block diagram of the GPS $\mathrm{L}_{5}$ PRN sequence generatortaken and modified from [11].

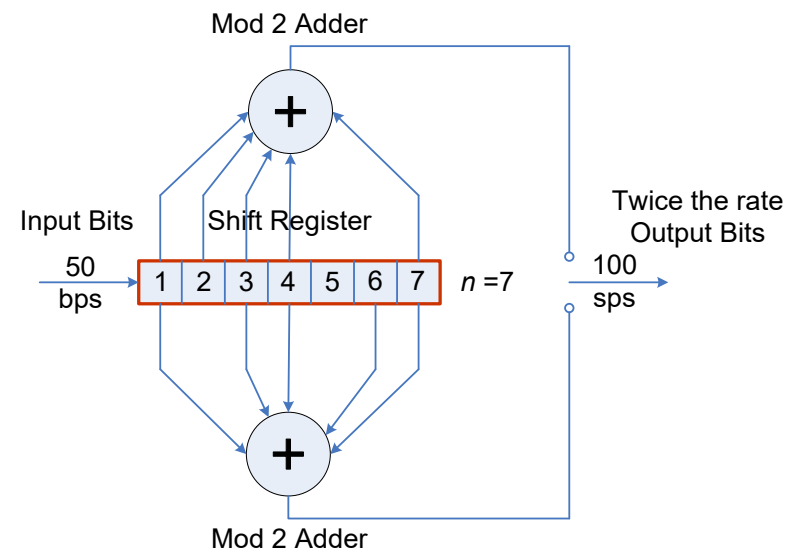

(d) A block diagram of the $r=1 / 2, n=7$, FEC sequence generator.

FIGURE 1: GPS L5 transmitter main components block diagram.

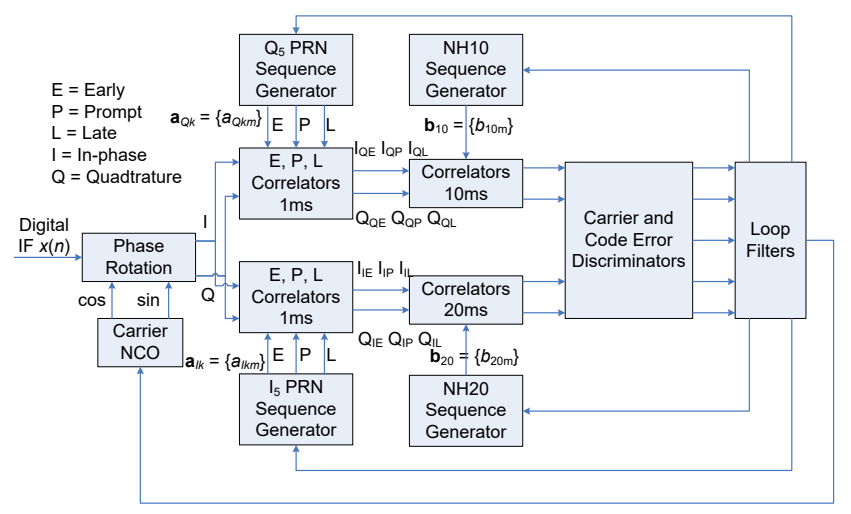

A block diagram of generic digital GPS $\mathrm{L}_{5}$ receiver channeltaken and modified from [12].

FIGURE 2: GPS L5 digital receiver main component block diagram.

$$
D=20 T
$$

where $\theta_{k}$ is the total phase and the explanation of the other components follows in order:

1. $a_{I k}\left(t_{k}\right)$ and $a_{Q k}\left(t_{k}\right)$ are the I and Q spreading codes at time $t_{k}$, which for convenience we assume that they are periodic with time period, $T$

2. $d_{k}\left(\left[t_{k}\right]_{D}\right)$ is the source symbol for satellite $k$ and spread by the code $a_{I k}\left(t_{k}\right)$ at symbol index $\left[t_{k}\right]_{D}$ (where $\lfloor z\rfloor$ denotes the greatest number less than or equal to $z$ ) and $D$ is the period when a data bit transition occurs and for the GPS case it is equal to $D$.

3. $f_{k}$ is the IF frequency which includes also the Doppler frequency of the $k$ th GPS satellite as observed from a GPS receiver and the index $k$ changes from $\{1 \cdots k\}$. Without loss of generality assume that the initial phase (not shown 
in the equation) is 0 .

4. $b_{10}\left(t_{k}\right)$ and $b_{20}\left(t_{k}\right)$ are respectively the respectively the 10 and 20 Newman Hofmann codes used on the data and pilot components at time $t_{k}$.

5. $T_{S}$ is the sampling period.

6. $\tau_{k}$ is the propagation delay between the $k^{\text {th }}$ GPS satellite and the GPS receiver under consideration.

A generic digital GPS $\mathrm{L}_{5}$ receiver channel is illustrated in Fig. 2. The digital IF signal $x(n)$ contains both the $\mathrm{I}$ and the $\mathrm{Q}$ components and residual Doppler frequency and channel noise. Initially the residual Doppler frequency is removed during the phase rotation phase. Next, the I and Q component of the baseband digital signal are first correlated with the early, prompt, and late replica of the $\mathrm{I}_{5}$ and $\mathrm{Q}_{5}$ sequences to determine the start of the GPS $\mathrm{L}_{5}$ PRN sequence; hence, the acquisition [11]. At the end of this process there will be 6 digital outputs for the I channel namely, $\mathrm{I}_{\mathrm{QE}}, \mathrm{I}_{\mathrm{QP}}, \mathrm{I}_{\mathrm{QL}}$, and $\mathrm{I}_{\mathrm{IE}}, \mathrm{I}_{\mathrm{IP}}, \mathrm{I}_{\mathrm{IL}}$ and 6 digital outputs for the $\mathrm{Q}$ channel namely, $\mathrm{Q}_{\mathrm{QE}}, \mathrm{Q}_{\mathrm{QP}}, \mathrm{Q}_{\mathrm{QL}}$ and $\mathrm{Q}_{\mathrm{IE}}$, QIP, Q $I L$ where the I and Q denote the channel index and the subscript index denote the PRN sequence replica. The six outputs of the first correlator are correlated with the NH10 sequence replica during a $10 \mathrm{~ms}$ period and the six outputs of the second correlator are correlated with the NH20 sequence replica during a $20 \mathrm{~ms}$ period. At the end of this process there are still going to be 12 correlation sums outputs: four early, four prompt, and four late. The four early and the four late correlation sum outputs are used code tracking whereas the four correlation prompt sums are used for acquisition, carrier tracking, and data demodulation.

\section{$3.1 \quad L_{5}$ Signal Acquisition}

The acquisition of the $\mathrm{I}_{5}$ and $\mathrm{Q}_{5}$ codes is very similar to the acquisition of the $\mathrm{L}_{1} \mathrm{C} / \mathrm{A}$ code. For this reason we can employ the full length correlation or partial length correlation; i.e., either the regular correlators or the narrowband correlators. Because the $\mathrm{I}_{5}$ channel contains data the $\mathrm{Q}_{5}$ channel does not contain data then it is easier to acquire the $\mathrm{Q}_{5}$ code first.

Yung et al. [11] suggest that joint $\mathrm{I}_{5}$ and $\mathrm{Q}_{5}$ acquisition has the potential to yield better performance in low SNRs; therefore, a joint $\mathrm{L}_{5}$ receiver has to potential to work indoors. But by the same token at low SNRs an $\mathrm{L}_{5}$ receiver would be more vulnerable to interference and jamming. Also by the same token for the same $S N R$ the joint $I_{5}$ and $Q_{5}$ acquisition would provide better interference and jamming resistance.

\section{2 $\boldsymbol{L}_{5}$ Signal Tracking}

A generic GPS $\mathrm{L}_{5}$ receiver can acquire and track the $\mathrm{L}_{5}$ signal and demodulate the data messages at least in three different ways: use $\mathrm{I}_{5}$ only to perform both signal tracking and data demodulation; use $\mathrm{Q}_{5}$ to track the $\mathrm{L}_{5}$ signal and $\mathrm{I}_{5}$ to perform the data demodulation; and use both $\mathrm{I}_{5}$ and $\mathrm{Q}_{5}$ to track the $\mathrm{L}_{5}$ signal and $\mathrm{I}_{5}$ to demodulate the data.

Since both the acquisition and tracking of the $\mathrm{L}_{5}$ signal is based on the sliding correlator approach that means that a generic $\mathrm{L}_{5}$ receiver is vulnerable to unintentional interference and jamming; especially with the types of jammers reported in the literature [9], [13]. Further acquisition algorithm and tracking details of a generic GPS $\mathrm{L}_{5}$ FFT and/or correlator type receiver are reported in [11].

\section{Jamming Signal Model}

Several jamming signal models are considered in the paper. These signals are communications signals which can be generated easily by means of analog or digital communications. Bastide el al [5] proposes three kids of interference signals: $\mathrm{CW}$ FM; DME $\backslash$ TACAN pulsed signals. Because there is a much larger set of inference signals than those proposed by Bastide el al [5] we propose to classify the jamming signals as: periodic, deterministic; aperiodic, deterministic; and noisy type signals or non-deterministic. This classification of jamming signals is much more general and is indented to provide a much broader set of jamming (or jammer) signals and at the same time to capture the essence of each group. However, we remind the reader that this approach is not the best approach to classify the jamming signals it is only a way that the authors intend to classify and represent the jamming signals.

\subsection{Periodic deterministic jamming signals}

A periodic deterministic signal consists of the following parameters: Jammer-to-Signal ratio, $\mathrm{J} \backslash \mathrm{S}$; frequency offset, $f_{\text {OFF }}$, with respect to the $\mathrm{L}_{5}$; period, $\mathrm{T}$; modulation type, MT; signal bandwidth, W.

There are several examples of periodic deterministic signals: CW signal; Amplitude modulated (AM) signal; FM signal; Phase Modulated (PM) signal; Pulse AM (PAM) signal; Pulse Position Modulated (PPM) signal; Pulse Width (or Duration) Modulated (PWM) signal; Pulse Code Modulated (PCM) signal. 


\subsection{Aperiodic deterministic jamming signals}

An aperiodic deterministic signal consists of the following parameters: $\mathrm{J} \backslash \mathrm{S}$, frequency offset, $f_{O F F}$, with respect to the $\mathrm{L}_{5}$; and signal bandwidth, W.

There are two good examples of aperiodic deterministic jamming signals: (a) any periodic deterministic signal in which the frequency offset is an irrational number; (b) a generalized frequency hopping jammer.

The second type of aperiodic deterministic jammer is more interesting because it is used in military radars [10]. A generalized frequency hopping jammer consists of one or more pulses being transmitted at different frequency slots (or bins). In general, the switching sequence from slot to slot is an encrypted algorithm and the frequency range occupied by all the possible bins or slots is as wide as possible [10]. If we were to simplify the model and consider the switching sequence from slot to slot as an aperiodic sequence then we have an aperiodic deterministic jamming signal.

\section{$4.3 \quad$ Noisy type or non-deterministic jamming signals}

The noisy type or non-deterministic jamming signal consists of the following parameters: $J \backslash S$; jamming signal bandwidth, W; autocorrelation function or power spectral density function.

These types of jammers are the most sever ones because they tend to copy the signal structure of one of the GPS signals and transmit the jamming signal at a higher power; and hence, jam the GPS signal [13]. According to Greton by implementing GAMES, GILS, GIANT, the question behind GPS reliability is a thing of the past [13].

First, implementing GAMES, GILS, GIANT is a very costly solution which for the purposes of everyday applications for the average user is out of question. Second, there is a large number of civilian GPS users that have no jamming protection at all. Therefore, all these users need some kind of protection. The maximum likelihood algorithm used for jointly estimating the GPS parameters presented in [14] which can be easily extend account for interference and jamming.

In the last few years, I and some of my colleagues have been working on a type of receiver which considers all the signals in the environment and then makes a decision about the signal detection based on a joint optimization function [14].

\section{Simulation Results}

First, it is not possible to consider all the jamming scenarios reported in the jamming signal section and a majority of these scenarios are reported in the literature anyway. However, the type of the jammer I would be most concerned with is the one which replicates any of the known $\mathrm{C} / \mathrm{A}$ codes for the $\mathrm{L}_{1}$ signal or the $\mathrm{PRN} \mathrm{I}_{5}$ or $\mathrm{Q}_{5}$ signals for the $\mathrm{L}_{5}$ code. These kinds of jammers can be easily built nowadays as suggested by [13].

However, the method of protection does not seem to be economically viable. Nevertheless, a GPS ML type of receiver which considers all the signals in the environment based on the model presented in [14] will almost always outperform a GPS CC type receiver.

\section{$5.1 \quad L_{1} C / A$ code acquisition simulation}

By treating all the GPS C/A code signals in the environment jointly, it is possible to greatly outperform the standard sliding correlator technique especially in situations where the GPS satellite signals are received with widely varying powers. For a simple numerical example, consider a simulated environment wherein four GPS satellites have been received at $-25,-23$, -20 and $10 \mathrm{~dB}$ signal to white noise power (SWNR). The received environment is modeled at complex baseband assuming the reception of a $1 \mathrm{~ms}, 1023$ chip Gold codes from 4 GPS satellites. For the purposes of this simulation the $4^{\text {th }}$ GPS satellite is considered as a jamming signal. We have also assumed that a standard deviation on the GPS receiver clock error is half of the chipping period, $T_{c}$.

The normalized cross correlation coefficient for the weakest signal, at $-25 \mathrm{~dB}$ is shown in Fig. 3(a) (top). The simple CrossCorrelator (CC) cannot detect the weakest signal at $-25 \mathrm{~dB}$ because the strongest signal at $10 \mathrm{~dB}$ can jam the weakest signal. In contrast the ML estimator (or function), shown in Fig. 3(a) (bottom), can detect the weakest signal most of the time. Because the strong GPS satellite codes have been cancelled, the ML estimator can still pick up a clear peak at the correct delay most of the time.

The normalized cross correlation coefficient for the second weakest signal, at $-23 \mathrm{~dB}$ is shown in Fig. 3(b) (top). Because of the strongest signal $10 \mathrm{~dB}$ or $33 \mathrm{~dB}$ stronger than the second weakest signal it is not possible for the simple cross correlator to detect this signal. Contrast this with the multiple ML estimator shown in Fig. 3(b) (bottom). Because the strong GPS satellite codes have been cancelled, the ML estimator can still pick up a clear peak at the correct delay. 

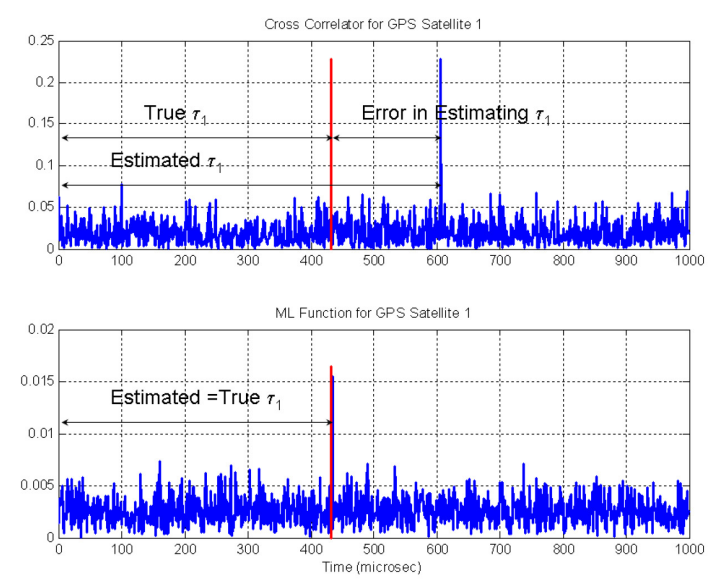

(a) The estimated and true $\tau_{1}$ using the $\mathrm{CC}$ (top) and ML function (bottom).


(b) The estimated and true $\tau_{2}$ using the CC (top) and ML function (bottom).
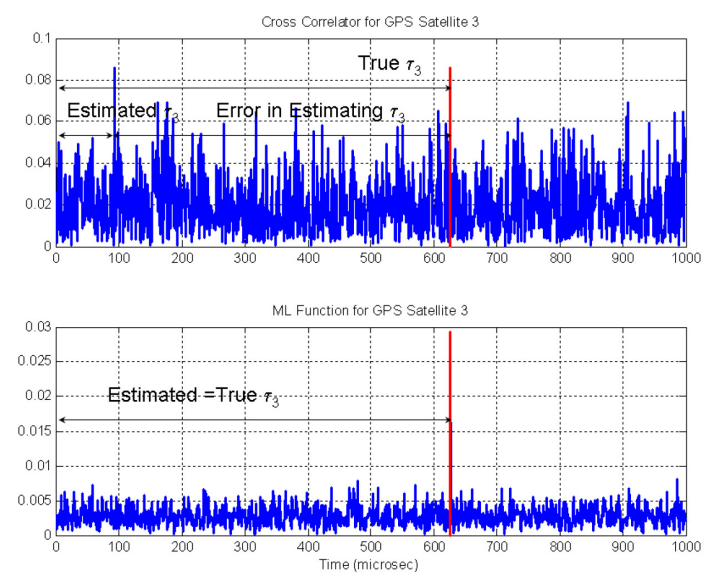

(c) The estimated and true $\tau_{3}$ using the CC (top) and ML function (bottom).
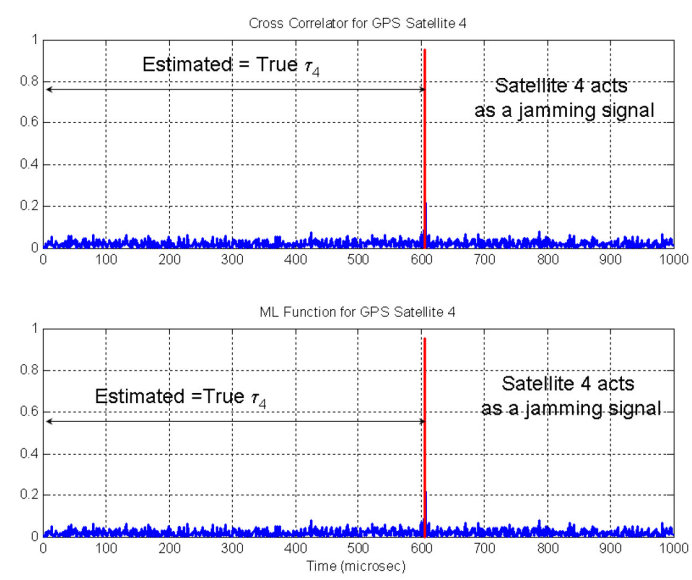

(d) The estimated and true $\tau_{4}$ using the CC (top) and ML function (bottom).

FIGURE 3: Four GPS satellite CC function (top) vs. ML function (bottom).

The normalized cross correlation coefficient for the second strongest signal, at $-20 \mathrm{~dB}$ is shown in Fig. 3(c) (top). Because of the strongest signal at $20 \mathrm{~dB}$ stronger than the second strongest signal it is not possible for the simple cross correlator to always detect this signal. Contrast this with the multiple user ML estimator shown in Fig. 3(c) (bottom). For this case the estimator can always pick up a clear peak at the correct delay all the time.

The normalized cross correlation coefficient for the strongest signal, at $10 \mathrm{~dB}$ is shown in Fig. 3(d) (top). Because this signal dominates all the other signals it is possible for the simple cross correlator to always detect this signal. Similarly, the ML estimator, shown in Fig. 3(d) (bottom), can always pick up a clear peak at the correct delay all the time.

This simple example clearly illustrates that if we were to build a jammer with signal one of the satellites signals and broadcast at $10 \mathrm{~dB}$ level able the noise floor than all the GPS receivers which use a simple $\mathrm{CC}$ for acquisition will be jammed. If however we were to use a ML estimator than the receiver will be able to detect all the GPS signals in the environment.

\section{$5.2 \quad L_{1} C / A$ code acquisition Monte Carlo simulation}

This same receiver's scenario was run over 100 Monte Carlo trials while randomly varying the thermal noise, delay offsets gold sequence seeds. The standard moving $\mathrm{CC}$ achieved a median absolute delay estimation error of \{300 27829010.04$\}$ microseconds and was never really able to detect the first weakest signal and rarely able to detect the second weakest 

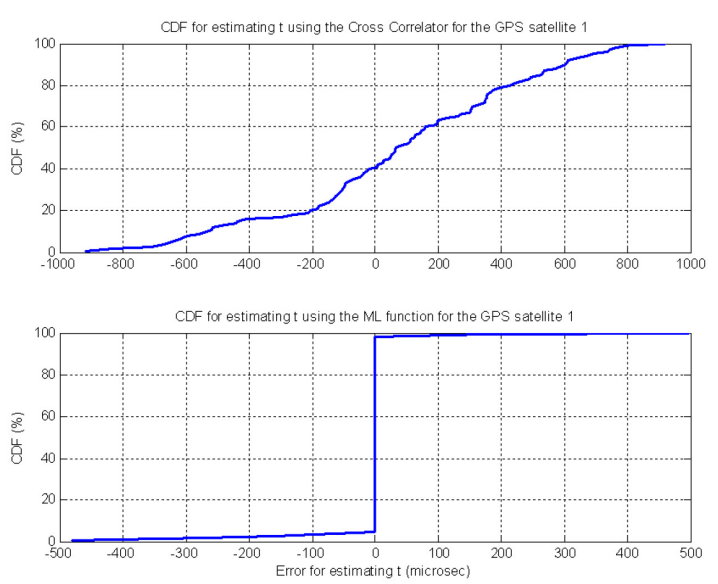

(a) The CDF of the estimated $\tau_{1}$ using the CC (top) vs ML function (bottom).
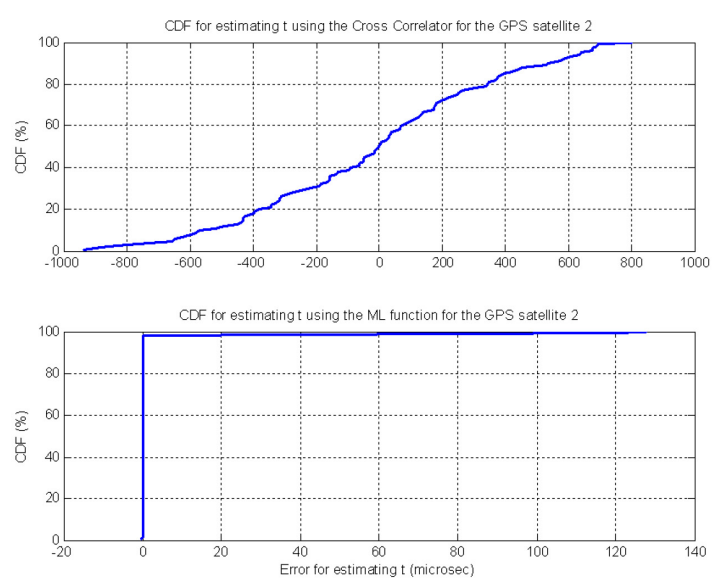

(b) The CDF of the estimated $\tau_{2}$ using the CC (top) and ML function (bottom).
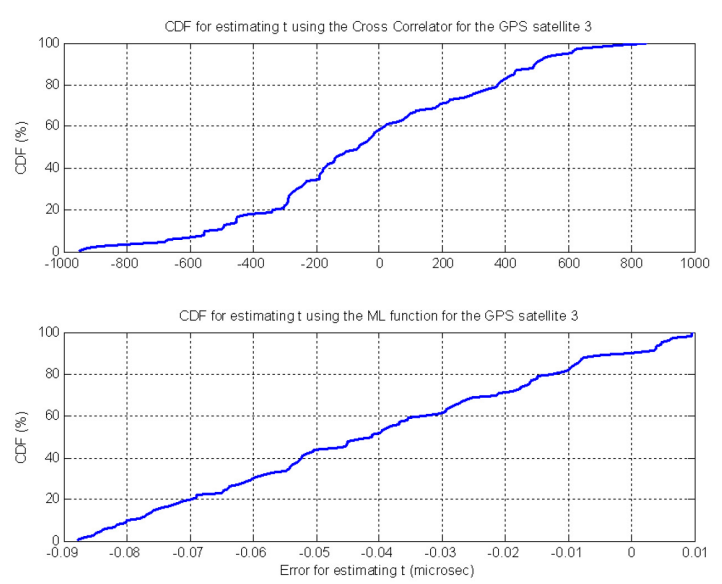

(c) The CDF of the estimated $\tau_{3}$ using the CC (top) and ML function (bottom).
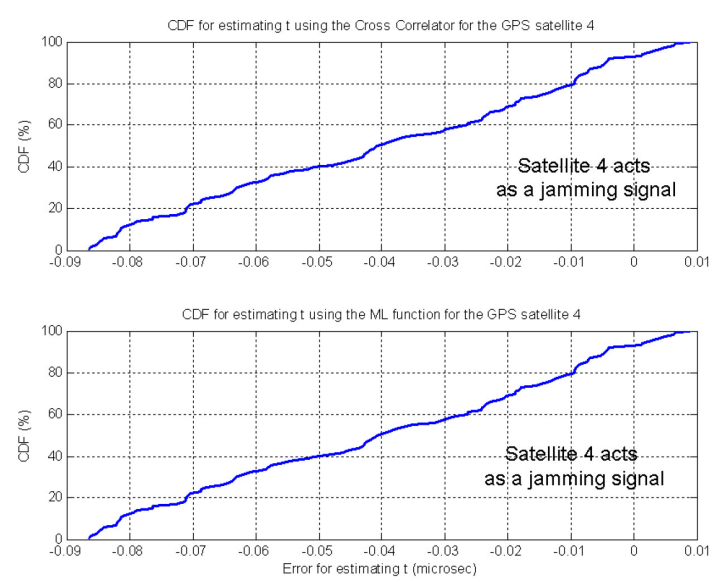

(d) The CDF of the estimated $\tau_{4}$ using the CC (top) and ML function (bottom).

FIGURE 4: Four GPS satellite CDF: CC function (top) vs. ML function (bottom).

signal. The ML estimator was successful in all 100 trials; however, it achieved a median absolute delay error of $\{46.2$ $42.74242 .2\}$ nanoseconds.

Figure 4(a) presents the CDF for estimated $\tau_{1}$ (i.e., the $1^{\text {st }}$ GPS satellite) using the CC (top) and the ML estimator (bottom). As shown in Fig. 4(a) (top) if the CC is used then there is a probability of $100 \%$ that the error for estimating $\tau_{1}$ will be from -900 to 900 microseconds. On the other hand, as shown in Fig. 4(a) (bottom), if the ML function is used then there is a probability of $98 \%$ that the error for estimating $\tau_{1}$ will be from -80 to 0 nanoseconds.

Figure $4(\mathrm{~b})$ presents the CDF for estimated $\tau_{2}$ (i.e., the $2^{\text {nd }}$ GPS satellite) using the CC (top) and the ML estimator (bottom). As shown in Fig. 4(b) if the CC is used then there is a probability of $99 \%$ that the error for estimating $\tau_{2}$ will be from -900 to 750 microseconds. On the other hand, as shown in Fig. 4(b) (bottom), if the ML function is used then there is a probability of $99.5 \%$ that the error for estimating $\tau_{2}$ will be from -115 to 80 nanoseconds.

Figure $4(\mathrm{c})$ presents the CDF for estimated $\tau_{3}$ (i.e., the $3^{\text {rd }}$ GPS satellite) using the CC (top) and the ML estimator. As shown in Fig. 4(c) (top), if the CC is used then there is a probability of $99 \%$ that the error for estimating $\tau_{3}$ will be from -900 to 830 microseconds. On the other hand, as shown in Fig. 4(c) (bottom), if the ML function is used then there is a $100 \%$ probability that both estimators achieve an error for estimating $\tau_{3}$ of -85 to 10 nanoseconds. 

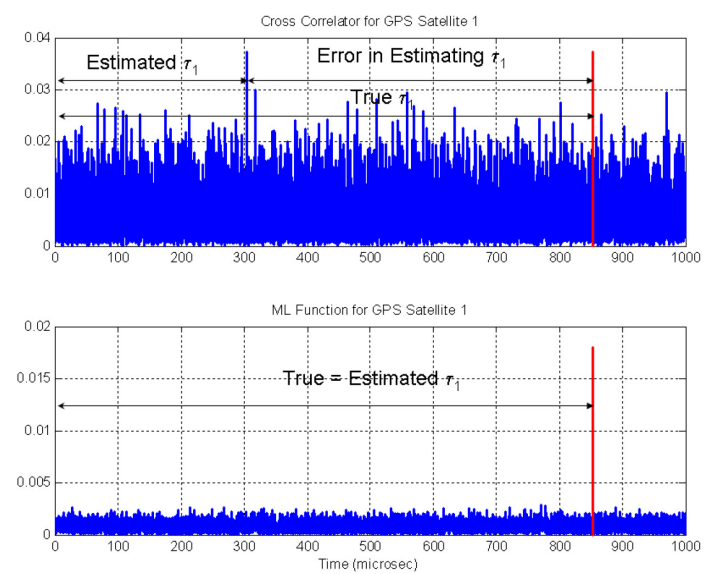

(a) The estimated and true $\tau_{1}$ using the $\mathrm{CC}$ (top) and ML function (bottom).
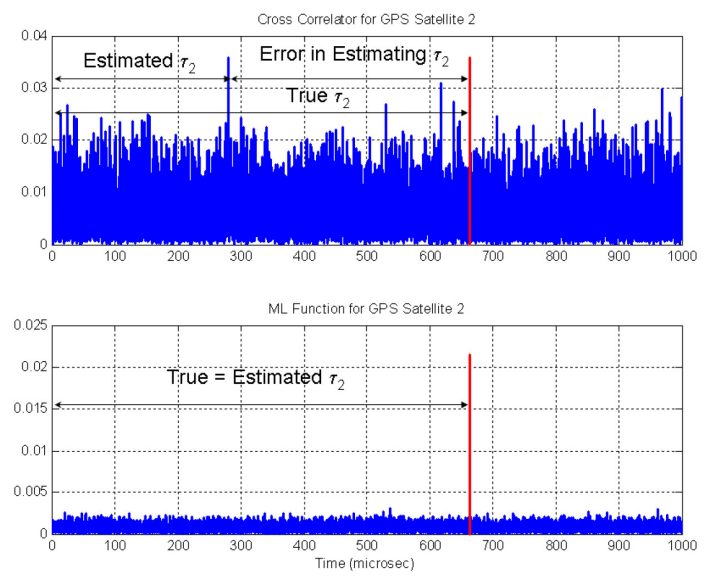

(b) The estimated and true $\tau_{2}$ using the CC (top) and ML function (bottom).
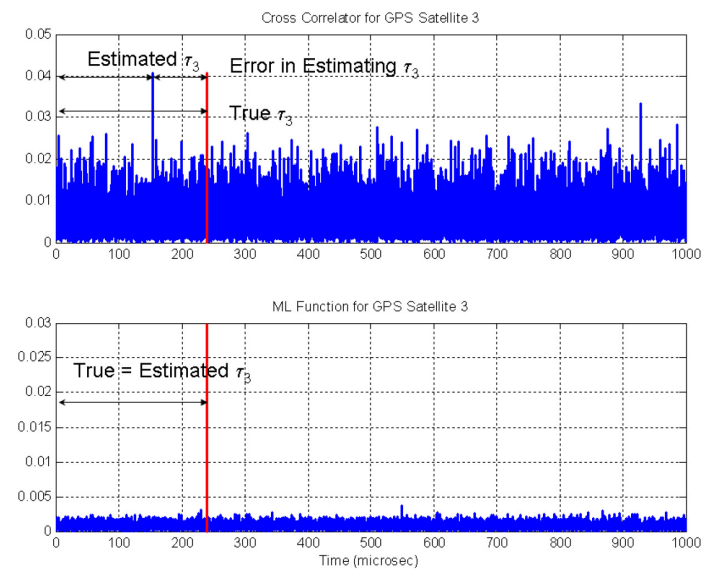

(c) The estimated and true $\tau_{3}$ using the CC (top) and ML function (bottom).


(d) The estimated and true $\tau_{4}$ using the CC (top) and ML function (bottom).

FIGURE 5: The cdf and pdf of a Lognormal, Rician, and Rayleigh fading channel for $\mu_{a}=0.6$ and $\sigma_{a}=0.0626$.

Figure $4(\mathrm{~d})$ presents the CDF for estimated $\tau_{4}$ (i.e., the $4^{\text {th }}$ GPS satellite (which acts as a jamming signal) using the CC (top) and the ML estimator (bottom). There is a $100 \%$ probability that both estimators achieve an error for estimating $\tau_{4}$ of -85 to 5 nanoseconds.

As indicated by the simulation results, the ML estimator is always superior to the standard CC. Therefore, we propose the maximum likelihood GPS receiver as a novel and optimum approach to process GPS signals.

\section{$5.3 \quad L_{5}$ PRN code acquisition simulation}

By treating all the $\mathrm{L}_{5}$ GPS signals in the environment jointly, it is possible to greatly outperform the standard sliding correlator technique especially in situations where the L $_{5}$ GPS satellite signals are received with widely varying powers. For a simple numerical example, consider a simulated environment wherein four satellites have been received at $-25,-23,-20$ and $10 \mathrm{~dB}$ SWNR. The received environment is modeled at complex baseband assuming the reception of a $1 \mathrm{~ms}, 10230$ chip Gold codes from $4 \mathrm{~L}_{5}$ GPS satellites. For the purposes of this simulation the $4^{\text {th }} \mathrm{L}_{5}$ GPS satellite is considered as a jamming signal. We have also assumed that a standard deviation on the GPS receiver clock error is half of the chipping period, $T_{c}$.

The normalized cross correlation coefficient for the weakest signal, at $-25 \mathrm{~dB}$ is shown in Fig. 5(a) (top). The simple CC cannot detect the weakest signal at $-25 \mathrm{~dB}$ because the strongest signal at $10 \mathrm{~dB}$ can jam the weakest signal. In contrast the ML estimator (or function), shown in Fig. 5(a) 
(bottom), can detect the weakest signal most of the time. Because the strong $\mathrm{L}_{5}$ GPS satellite codes have been cancelled, the ML estimator can still pick up a clear peak at the correct delay most of the time.

The normalized cross correlation coefficient for the weakest signal, at $-23 \mathrm{~dB}$ is shown in Fig. 5(b) (top). The simple $\mathrm{CC}$ cannot detect the weakest signal at $-23 \mathrm{~dB}$ because the strongest signal at $10 \mathrm{~dB}$ can jam the weakest signal. In contrast the ML estimator (or function), shown in Fig. 5(b) (bottom), can detect the weakest signal most of the time. Because the strong L $_{5}$ GPS satellite codes have been cancelled, the ML estimator can still pick up a clear peak at the correct delay most of the time.

The normalized cross correlation coefficient for the second strongest signal, at $-20 \mathrm{~dB}$ is shown in Fig. 5(c) (top). Because of the strongest signal at $20 \mathrm{~dB}$ stronger than the second strongest signal it is not possible for the simple cross correlator to always detect this $\mathrm{L}_{5}$ GPS signal. Contrast this with the multiple user ML estimator shown in Fig. 5(c) (bottom). For this case the estimator can always pick up a clear peak at the correct delay all the time.

The normalized cross correlation coefficient for the strongest signal, at $10 \mathrm{~dB}$ is shown in Fig. 5(d) (top). Because this signal dominates all the other signals it is possible for the simple cross correlator to always detect this L $_{5}$ GPS signal. Similarly, the ML estimator, shown in Fig. 5(d) (bottom), can always pick up a clear peak at the correct delay all the time.

This simple example clearly illustrates that if we were to build a jammer with signal one of the satellite signals and broadcast at $10 \mathrm{~dB}$ level above the noise floor than all the GPS receivers which use a simple $\mathrm{CC}$ for acquisition will be jammed. If however we were to use a ML estimator than the receiver will be able to detect all the GPS signals in the environment.

Due to the immense amount of time required to run these simulations we have not provided the Monte-Carlo simulation data for the $\mathrm{L}_{5}$ GPS signal.

\section{Conclusions}

It appears that the community agrees that there are several advantages of the new GPS signal. It has stronger power compared to the civilian signals transmitted at the $\mathrm{L}_{1}=1575.42$ $\mathrm{MHz}$ and $\mathrm{L}_{2}=1227.6 \mathrm{MHz}$ [2]. Unlike the BPSK modulation scheme used for the civilian signals transmitted at the $\mathrm{L}_{1}$ and $\mathrm{L}_{2}$, the modulation scheme used for the new $\mathrm{L}_{5}$ signal is QPSK.
The chipping frequency for the new signal is 10 times higher than chipping frequency used for the signals in the $L_{1}$ and $L_{2}$. It appears to offer better multipath and narrowband interference resistance [7]. For these reasons and other reasons reported in the literature the new $\mathrm{L}_{5}$ GPS signal is more suitable for a variety of civilian applications in addition to the $\mathrm{L}_{1}$ and $\mathrm{L}_{2}$ signals.

However, the majority of vendors are still using correlator type receivers. As shown by the simulation results of this paper and other previous publications a sliding correlator type of receiver used to acquire and track the $\mathrm{C} / \mathrm{A}$ code or $\mathrm{L}_{5}$ code can be easily jammed by a similar C/A code replica or $\mathrm{L}_{5}$ code replica at power levels $10 \mathrm{~dB}$ or higher above noise power. GAMES, GILS, and GIANT are not the most economically viable solutions in the market to handle GPS protection of the civilian receivers.

A maximum likelihood GPS receiver which considers all the signals in the environment can be used to acquire and track successfully the $\mathrm{C} / \mathrm{A}$ and the $\mathrm{L}_{5}$ GPS signals when the jamming signal power are in the range or $10 \mathrm{~dB}$ or higher above the noise power.

In summary, the direction of designing acquisition and tracking receivers for future $\mathrm{C} / \mathrm{A} \mathrm{L}_{1}$ code or $\mathrm{L}_{5}$ GPS signals should go towards joint signal acquisition and tracking. There are three main challenges with these types of receivers: algorithm complexity, computational power, and test with real data. All these remain to be studied and analyzes further in the future. I think it is going to be a breakthrough in the state of the art of GPS receivers when these algorithms are ultimately implemented and a lot more work is required to arrive at that point which some of it is going to be published in future publications.

\section{Acknowledgement}

This work was supported by California State Polytechnic University (Cal Poly), Pomona, CA in 2006.

\section{References}

[1] I. Progri, "GPS L5 signal acquisition and tracking under unintentional interference or jamming," in Proc. IONNTM 2006, Monterey, CA, pp. 112-121, Jan. 2006, http://giftet.com/Progri/Progri_2006_01_01_ION_NTM.p df. 
[2] J.J. Spilker Jr., A.J. Van Dierendonck, "Proposed new L5 civil GPS codes," Navigation, vol. 48, nr 3. pp. 135-143, fall 2001, DOI: http://doi.org/10.1002/j.21614296.2001.tb00237.x.

[3] M. Tran, C. Hegarty, "Receiver algorithms for the new civil GPS signals," in Proc. ION-NTM 2002, San Diego, CA, pp. 778-789, Jan. 2002.

[4] L. Ries, L. Lestarquit, E. Armengou-Miret, F. Legrand, W. Vigneau, C. Bourga, P. Erhard, J.L. Issler, "A software simulation tool for GNSS2 BOC signals analysis," in Proc. ION-GPS 2002, Portland, OR, pp. 2225-2239, Sep. 2002.

[5] F. Bastide, C. Macabiau, D. Akos, B. Roturier, "Assessment of L5 receiver performance in presence of interference using a realistic receiver simulator," in Proc. $I O N-G P S / G N S S$ 2003, Portland, OR, pp. 142-152, Sept. 2003.

[6] C. Macabiau, L. Ries, F. Bastide, J-L Issler, "GPS L5 receiver implementation issues," in Proc. ION-GPS/GNSS 2003, Portland, OR, pp. 153-164, Sept. 2003.

[7] C. Hegarty, M. Tran, A.J. Van Dierendonck, "Acquisition algorithms for the GPS L5 signal," in Proc. IONGPS/GNSS 2003, Portland, OR, pp. 165-177, Sept. 2003.

[8] C. Hegarty, M. Tran, J.W. Betz, "Multipath performance of the new GNSS signals," in Proc. ION-NTM 2004, San Diego, CA, pp. 333-342, Jan. 2004.

[9] B. Zheng, G. Lachapelle, "Acquisition schemes for a GPS L5 software receiver," in Proc. ION-GNSS 2004, Portland, OR, pp. 1035-1040, Sept. 2004.

[10] J-L. Issler, L. Ries, J-M. Bourgeade, L. Lestarquit, C. Macabiau, "Probabilistic approach of frequency diversity as interference mitigation means," in Proc. ION-GNSS 2004, Portland, OR, pp. 2136-2145, Sept. 2004.

[11]C. Yung, C. Hegarty, M. Tran, “Acquisition of the GPS L5 signal using coherent combining of I5 and Q5," in Proc. ION-GNSS 2004, Portland, OR, pp. 2184-2195, Sept. 2004.

[12]I.F. Progri, W.R. Michalson, M.C. Bromberg, "A DSSS/CDMA/FDMA indoor geolocation system," in Proc. ION-GPS, Portland, OR, pp. 155-164, Sep. 2002.

[13]G. Gerton, "Protecting the Global Positioning System," IEEE Aero. \& Elect. Sys. Mag., vol. 20, nr. 11, pp. 3-8, Nov. 2005, DOI: https://doi.org/10.1109/MAES.2005.1576067.

[14] I. Progri, M.C. Bromberg, W.R. Michalson, "Maximum likelihood GPS parameter estimation," Navigation, vol.
52, no. 4, pp. 229-238, winter 2005-2006, DOI: http://doi.org/10.1002/j.2161-4296.2005.tb00365.x, URL: http://giftet.com/Progri/Progri_2005_winter_Navigation.p df.

[15] I. Progri, M.C. Bromberg, W.R. Michalson, J. Wang, “A theoretical survey of the spreading modulation of the new GPS signals (L1C, L2C, and L5)," J. Geoloc. GeoInf. Geoint., vol. 2017, pp. 70-82, DOI: http://doi.org/10.18610/JG3.2017.071601.

[16] I. Progri, “On generalized multi-dimensional geolocation modulation waveforms," in Proc. IEEE/ION-PLANS 2012, Myrtle Beach, SC, pp. 919-951, Apr. 2012, DOI: http://doi.org/10.1109/PLANS.2012.6236835.

[17]I. Progri, "VBOC1 $(\alpha)$ generalized multidimensional geolocation modulation waveforms," J. Geoloc. GeoInf. Geoint., vol. 2015, pp. 19-31, Nov. 2015, DOI: http://doi.org/10.18610/JG3.2015.082101.

[18] I. Progri, "VBOC1( $\alpha$ ) ACF pure signal optimization," $J$. Geoloc. GeoInf. Geoint., vol. 2015, pp. 32-43, Nov. 2015, DOI: http://doi.org/10.18610/JG3.2015.082102.

[19] I. Progri, "VBOC $2(\alpha, 1-\alpha)$ generalized multidimensional geolocation modulation waveforms," J. Geoloc. GeoInf. Geoint., vol. 2015, pp. 44-56, Nov. 2015, DOI: http://doi.org/10.18610/JG3.2015.082103.

[20] I. Progri, "VBOC2 $(\alpha, 1-\alpha)$ ACF pure signal optimization," J. Geoloc. GeoInf. Geoint., vol. 2015, pp. 56-69, Nov. 2015, DOI: http://doi.org/10.18610/JG3.2015.082104.

[21]I. Progri, "VBOC $1(\alpha)$ and $\operatorname{VBOC} 2(\alpha, 1-\alpha)$ generalized multidimensional geolocation modulation waveforms - technical report," J. Geoloc. GeoInf. Geoint., vol. 2015, pp. 70-82, Nov. 2015, DOI: http://doi.org/10.18610/JG3.2015.082105.

[22] I. Progri, Indoor Geolocation Systems-Theory and Applications. Vol. I, $1^{\text {st }}$ ed., Worcester, MA: Giftet Inc., $\sim 800$ pp., $\sim 2017$ (not yet available in print).

[23] I. Progri, W.R. Michalson, "An alternative approach to multipath and near-far problem for indoor geolocation systems," in Proc. $14^{\text {th }}$ Inter. Tech. Mtg. Sat. Div. ION (ION GPS 2001), Salt Lake City, UT, pp. 1434-1443, Sep. 2001.

[24]I. Progri, W.R. Michalson, J. Wang, M.C. Bromberg, R.J. Duckworth, "Requirements of a C-CDMA pseudolite indoor geolocation system," J. Geol. Geoinfo. Geointel., vol. 2017, article ID 2017071605, 5 pg., Nov. 2017. DOI: 
http://doi.org/10.18610/JG3.2017.071605, http://www.giftet.com/JG3/2017/071605.pdf.

[25] I. Progri, W.R. Michalson, J. Wang, M.C. Bromberg, "Indoor geolocation using FCDMA pseudolites: signal

i Signal structure is the arrangement of and relations between the parts or elements of the signal.

ii Signal design is the plan or drawing produced to show the look and function or workings of the signal structure before it is produced and transmitted from structure $^{\mathrm{v}}$ and performance analysis," Navigation, vol. 54, no. 3, pp. 242-256, fall 2007, DOI: http://doi.org/10.1002/j.2161-4296.2007.tb00407.x, http://giftet.com/Progri/Progri_2007_fall_Navigation.pdf.

the satellite or transmitter.

iii In this paper we offer a significant improvement on the notation of the received signal in contrast to [1].

iv Ditto

v Ditto (i) 